\title{
Threonine/Tyrosine Protein Kinase
}

National Cancer Institute

\section{Source}

National Cancer Institute. Threonine/Tyrosine Protein Kinase. NCI Thesaurus. Code C18152.

Class of enzymes that phosphorylate protein substrates at tyrosine or threonine residues. 